\title{
Continuity: The relation between concept image and procedural understanding
}

\author{
Meltem Coşkun ${ }^{1, *}$, and Necla Turanl1 ${ }^{1}$ \\ ${ }^{1}$ Department of Mathematics and Science Education, Hacettepe University, Ankara, Turkey
}

\begin{abstract}
The current study aims to examine the relation between concept images and procedural understanding of prospective Mathematics teachers (PMTs) related to 'continuity' in Math. The participants $(n=9)$ study Mathematics Education at a state university during spring 2018; they are third, fourth and fifth grade students. The data come from interviews and open-ended tests developed by the researchers. More specifically, the data of concept images were collected through interviews and the data of procedural understanding were gathered via open-ended tests. The methodological approach used in the current study is descriptive analysis. The results of the study revealed that in addition to the presence of the PMTs whose understanding of concept images and procedural understanding are in accordance with each other, there are those whose understanding of these two concepts are not compatible with each other. Moreover, it has been observed that while PMTs who hold a concordant understanding between concept image and procedural understanding tend to express the concept of 'continuity' as rooted in their images, PMTs who have an incompatible understanding between these two concepts tend to provide formal definitions for the notion of 'continuity', as different from their own images.
\end{abstract}

\section{Introduction}

The concept of 'continuity' is one of the core topics in the field of Math. This concept starts to be taught at high school years and continue to be explored in more details at undergraduate level. Majority of students taking Mathematics course encounter the notion of 'continuity'. However, it is of critical importance to understand how students in the Faculty of Education, more specifically, Mathematics Education majors perceive the concept as with the background knowledge they acquire in undergraduate years, they become the teachers who are likely to shape their students' minds in the development of the concept of 'continuity'. In this sense, it is critical to recognize early on if any inconsistencies occur between what prospective teachers develop as 'continuity' in their minds and what is represented as 'continuity' in Math. Moreover, if any incongruence exists, it is significant to uncover how these inconsistencies develop in order to better understand images created for the concept of 'continuity'. The concept image consists of all the cognitive structure in the individual's mind, associated with a given concept [3]. In

\footnotetext{
* Corresponding author: meltemcoskun@hacettepe.edu.tr
} 
this regard, cognitive structures of prospective teachers related to the concept of 'continuity' determine the images formed for the concept. In addition to concept images, prospective Mathematics teachers (henceforth PMTs) are supposed to be proficient in procedural understanding. Thus, how PMTs make use of features of 'continuity' and how they reflect these in their own mathematical operations are important in that it tells us how they develop procedural understanding. Against this background, the present study set out to explore the relationship between PMTs' concept images and procedural understanding related to 'continuity' in Math.

\section{Method}

The current study is conducted as a case study, one of qualitative research designs. The core aim of case studies is to provide factual evidence from a specific example [5]. The data was analyzed through descriptive analysis, one of qualitative data analysis methods.

\subsection{Participants}

The participants of the study were nine prospective teachers majoring in Mathematics Education at a state university in Ankara during the spring term of 2017-2018 academic year. They were third, fourth and fifth grade students.

\subsection{Data collection}

The data on which this study is based come from interviews and open-ended tests developed by the researchers. The content of the interviews consisted of five questions, which follow as such: (1) What comes to you mind when you hear the word continuity? (2) What evokes in your mind with the concept of continuity in Math? (3) Were it not for continuity, which mathematical operations would not you be able to perform? (4) What kind of figure, graphic, picture, explanation can you use for the concept of continuity? (5) What can be the equivalent of the concept of continuity in real life? As for the open-ended test, eight operational questions targeting at the concept of continuity in different spaces (i.e., $\mathrm{R}, \mathrm{R}^{2}$, metric, topological spaces) were directed to the participants. The data for concept images were collected through interviews and the data of procedural understanding were gathered via open-ended tests.

\subsection{Application process}

The open-ended tests aiming at exploring the procedural understanding of nine PMTs were conducted in a classroom setting. The practice took approximately one hour. Following this, individual interviews with the PMTs were performed in order to uncover their concept images on the matter at hand. All sessions were audio-recorded with the consent of the participants, who gave their informed consent forms to participate willingly in the study.

\subsection{Data analysis}

The data for concept images and procedural understanding of the PMTs were analyzed through content analysis and descriptive analysis, respectively. The relationship between the concept images and procedural understanding of the PMTs was subjected to descriptive analysis. 


\section{Findings}

One-on-one interviews were conducted with the PMTs in order to uncover their concept images on 'continuity' and open-ended tests were used to describe their procedural understanding on the matter at hand. Based on the yielded results, the concept images and procedural understanding of the PMTs on the notion of 'continuity' were illustrated: In what follows, results drawing attention to the right combination of concept images and procedural understanding were provided.

Participants were directed to five questions, aiming at their concept images on 'continuity'. The responses provided by the PMTs were put under nine categories, namely (1) continuing, (2) use of definitions, (3) types of 'continuity', (4) limit, (5) derivative, (6) integral, (7) graphic, (8) concrete expression, and (9) abstract expression. Interestingly, the category of 'continuing' was present in all of the answers given by the participants.

PMT1: "I think of something that is not interrupted, that is continuous."

PMT2: "I think of an uninterrupted function, that is, a continuous one without any barriers."

PMT3: "The 'continuity' in Math is something like without split. For example, like a rope, which continues straightforwardly."

PMT4: "It is continuous if we can draw without lifting our hands."

PMT5: "It means that something is continuous, nonstop and uninterrupted."

PMT6: "There is no break; in other words, it performs as it is expected to be."

PMT7: "It is curves that we draw without lifting our hands."

PMT8: "It is something that continues."

PMT9: "It means every uninterrupted thing we do in our lives."

In the category of 'continuing', PMTs describe the concept of 'continuity' with the expressions such as 'drawing without lifting the hand', 'continuing straightforwardly', 'being uninterrupted', and 'nonbeing of break-off'.

Eight questions were directed to the PMTs in order to investigate their procedural understanding. The first seven questions were related to 'continuity' in $\mathrm{R}, \mathrm{R}^{2}$, metric, topological spaces, and the last question was an individual question, which followed as such: 'Please write down an example of continuous and discontinuous function. Draw a graphic of these functions.' Based on the accuracy of the responses and the proficiency of the use of mathematical rules necessary for mathematical symbols and solutions of the problems, procedural understanding of the PMTs were evaluated under three categories: (1) poor, (2) average, and (3) good.

Once the identification of concept images and procedural understanding on the concept of 'continuity' was done, the relationship between these two concepts was examined. It was observed that there were PMTs who had a compatible understanding between concept images and procedural understanding as well as there were those whose understanding of these two concepts did not comply with each other.

It was demonstrated that those PMTs with a congruent view of the related concepts (i.e., concept image and procedural understanding) tended to express their understanding of 'continuity' in their own images when they were engaged in the mathematical operations:

Question 5 in the open-ended test follows as such: Please examine the 'continuity' of the function described as $f: R \rightarrow R, f(x)=x^{2}$. The response given by the PMT1 is presented below in Figure 1:

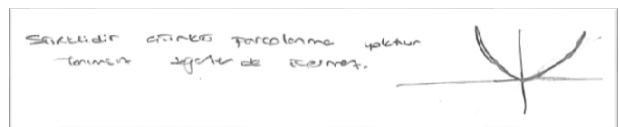

Fig. 1. The response PMT1 provided for question 5. 
As seen in Figure 1, PMT1 examines the 'continuity' of the related function in accordance with the image of itself. She mirrors what is presented as 'uninterrupted, continuous' in her image in the mathematical operation.

It has been observed that PMTs who had an incompatible understanding of concept image and procedural understanding tended to provide formal definitions for the concept of 'continuity', as different from their own images:

The answer provided by the PMT6 for the question 5 in the open-ended test is shown in Figure 2:

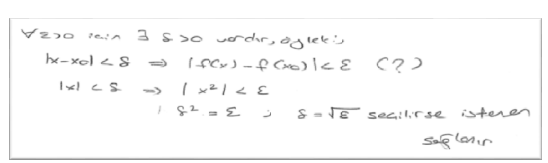

Fig. 2. The response PMT6 provided for question 5.

As it is visible in Figure 2, PMT6 examines the 'continuity' of the related function different from its image. The notion of 'nonbeing of break-off' in her image is not represented in the current operation. On the contrary, she performs mathematical operation through $\varepsilon-\delta$, one of the features of 'continuity' in Math.

Question 6 in the open-ended test is as following: A regular $\tau$ topology is present on set $R$. Accordingly, the function of $f:(R, \tau) \rightarrow(R, \tau)$ is described as $f(x)=|x|(\forall \in R)$. Please demonstrate the 'continuity' of function $f$. The answer given by the PMT8 is presented in Figure 3:

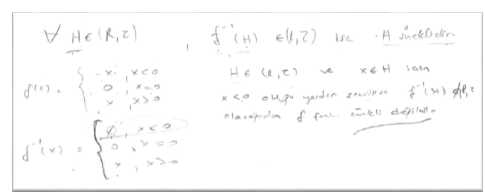

Fig. 3. The response PMT8 provided for question 6.

As can be seen in Figure 3, PMT8 interprets the 'continuity' of the related function different from its image itself. The notion of 'continuing' present in her image is not reflected in the mathematical operation. Interestingly, she makes use of the fact that each open set in second space is also open in the first space under the inverse image of the function, which is one of the core characteristics of 'continuity'.

Question 8 in the open-ended test follows as such: 'Please write down an example of continuous and discontinuous function. Draw a graphic of these functions.' In the current question, while PMTs were quite good at drawing a continuous function available in their images, they tended to display difficulties with the example of discontinuous function.

\section{Conclusions and suggestions}

The current study set out to investigate the relation between PMTs' concept images and procedural understanding related to 'continuity' in Math. To achieve this goal, PMTs were interviewed in order to uncover their concept images for the concept of 'continuity' and they were exposed to open-ended tests to better understand their procedural understanding on the matter at hand. Based on the yielded findings, the concept images and procedural understanding of the PMTs on the notion of 'continuity' were taken together. The results of the study revealed that although there are PMTs who have a congruent understanding between concept images and procedural understanding, there are also those whose understanding of these two concepts do not coexist in accord with each other. While PMTs with a compatible understanding of the related concepts (i.e., concept image and procedural 
understanding) are likely to express their understanding of continuity in their own images during the mathematical operations, PMTs with an incompatible understanding between these two concepts tend to provide formal definitions for the notion of 'continuity', as different from their own images. The study demonstrated that PMTs who relate the concept of 'continuity' to certain actions such as 'nonbeing of break-off', 'drawing without lifting the hand', and 'being uninterrupted' tend to reflect these concepts in their procedural understanding as well. Note that the study also shows how the concept of 'continuity' is not fully grasped but operations on the concept still can be achieved. This finding is in line with [1] and [4] studies, in which students were having difficulty in understanding the concepts but still perform the operations successfully. Moreover, the current finding is in comply with what [2] have found: 'students are not fully aware of what mathematical concepts truly are and what mathematics really means.' In this regard, it is important for the students to learn the concepts of the difficult topics but not the algorithmic calculus [2]. It is worth noting that PMTs holding a different procedural understanding from their images tend to examine the 'continuity' of a function through formal definitions on continuity, especially the definition accompanied with limit, the definition of $\varepsilon-\delta$ and lastly with the definition that each open set in second space is also open in the first space under the inverse image of the function. PMTs have not mentioned a continuous function with break-offs or interruptions although they could define the concept of 'continuity' with the formal definitions. To illustrate, there was no reference to a continuous function like $1 / x, x \neq 0$, in which break-off occurs or the hand is lifted during the process. It might be concluded that the different nature of the procedural understanding of the PMTs from their concept images can be attributed to the fact that teachers know what to expect from their students in question-answer episodes and thus try to provide a response for the related question.

The following suggestions can be offered to the researchers:

- Assessment instruments should not be used only to explore procedural understanding of prospective teachers, but they should also target at their concept images. It is highly important to detect and fix possible flaws in concept images as they might adversely affect their future teaching in their professional lives.

- As there are chances that students have flaws in their concept images, PMTs should be trained to better recognize concept images of their prospective students.

\section{References}

1. G. Harel, SSM, 89, 1, 40-57 (1989).

2. Y. Soylu, S. Aydın, EUJEF, 8, 2, 83-95 (2006).

3. D. Tall, S. Vinner, Educational Studies in Mathematics, 12, 151-169 (1981).

4. T. Wang, CEE, 23, 4, 236-241 (1989).

5. A. Yıldırım, H. Şimşek, Sosyal bilimlerde nitel araştırma yöntemleri (2016). 